\title{
The prognostic value of multivoxel magnetic resonance spectroscopy determined metabolite levels in white and grey matter brain tissue for adverse outcome in term newborns following perinatal asphyxia
}

\author{
Pieter Jan van Doormaal • Linda C. Meiners • \\ Hendrik J. ter Horst • Christa N. van der Veere • \\ Paul E. Sijens
}

Received: 8 July 2011 /Revised: 6 September 2011 / Accepted: 6 October 2011 /Published online: 7 November 2011

(C) The Author(s) 2011. This article is published with open access at Springerlink.com

\begin{abstract}
Objective Magnetic resonance spectroscopy can identify brain metabolic changes in perinatal asphyxia by providing ratios of metabolites, such as choline (Cho), creatine $(\mathrm{Cr})$, $\mathrm{N}$-acetyl aspartate (NAA) and lactate (Lact) [Cho/Cr, Lact/ NAA, etc.]. The purpose of this study was to quantify the separate white and grey matter metabolites in a slab cranial to the ventricles and relate these to the outcome.

Methods A standard 2D-chemical shift imaging protocol was used for measuring a transverse volume of interest located cranial to the ventricles allowing for direct comparison of the metabolites in white and grey matter brain tissue in 24 term asphyxiated newborns aged 3 to 16 days.

Results Cho, NAA and Lact showed significant differences between four subgroups of asphyxiated infants with more and less favourable outcomes. High levels of Cho and Lact
\end{abstract}

P. J. van Doormaal · H. J. ter Horst • C. N. van der Veere Department of Pediatrics, Division of Neonatology, University

Medical Center Groningen and University of Groningen,

Hanzeplein 1,

9713 GZ, Groningen, The Netherlands

L. C. Meiners • P. E. Sijens $(\bowtie)$

Department of Radiology, University Medical Center Groningen and University of Groningen,

Hanzeplein 1,

9713 GZ, Groningen, The Netherlands

e-mail: p.e.sijens@umcg.nl

Present Address:

P. J. van Doormaal

Department of Radiology, Meander Medical Center Amersfoort,

PO Box 1502, 3800 BM, Amersfoort, the Netherlands in the grey matter differentiated non-survivors from survivors ( $P=0.003$ and $P=0.017$, respectively).

Conclusion In perinatal asphyxia the levels of Cho, NAA and Lact in both white and grey matter brain tissue are affected. The levels of Cho and Lact measured in the grey matter are the most indicative of survival. It is therefore advised to include grey matter brain tissue in the region of interest examined by multivoxel MR spectroscopy.

Key Points

- Magnetic resonance spectroscopy can identify brain metabolic changes in perinatal asphyxia.

- Choline and lactate levels in grey matter seem the best indicators of survival.

- Both grey and white matter should be examined during spectroscopy for perinatal asphyxia.

Keywords Asphyxia $\cdot$ Encephalopathy $\cdot$ Magnetic resonance spectroscopy $\cdot$ Metabolism

\section{Introduction}

Standard clinical information used to determine prognosis in perinatal asphyxia, including Apgar scores, neurological information, evoked potentials, electroencephalography, cerebral blood flow and neuro-imaging, do not always reliably predict neurological outcome. In the past decades there have been numerous scientific studies indicating the prognostic utility of proton magnetic resonance spectroscopy (MRS) for prediction of outcome in infants with neonatal encephalopathy (NE). Thirty-two studies (860 infants with $\mathrm{NE}$ ) were included in a recent meta-analysis [1]. In the neonatal period (days 1-30) the metabolite ratio of lactate to 
$\mathrm{N}$-acetyl aspartate (Lact/NAA), most frequently measured in the basal ganglia and thalamus region where abnormalities are often seen on MRI [2], was the best biomarker for predicting adverse outcome following NE [1]. Changes in brain metabolism following asphyxia have generally been assessed in terms of ratios of metabolites such as choline (Cho)/ creatine (Cr), NAA/Cr and Lact/NAA [3-14]. In such results it is not possible to differentiate if, for example, an increase of the Lact/NAA ratio is caused by an increase of Lact or a decrease of NAA.

In the scarce quantitative MRS studies of asphyxiated infants with severe or fatal outcomes, decreases in the concentrations of Cho and NAA were found [15-17] and Lact concentration increases [15, 17]. In one of these studies significant $\mathrm{Cr}$ decreases were observed as well [16]. Another quantitative MRS study using a short echo time (TE), revealed decreases in the concentrations of NAA and glutamate/glutamine, and Lact concentration increases in infants with perinatal asphyxia compared to nonasphyxiated babies [18]. So far, there has been just one study providing a thorough comparison of the results obtained in white and grey matter brain tissue [16]. In a slab positioned at the level of the basal ganglia, a low NAA turned out to be the best indicator of unfavourable outcome both in white and grey matter structures [16].

The purpose of this study was quantitative comparison of the separate MRS detected metabolites at a level of the brain not yet studied in infants with asphyxia, a transverse slab cranial to the ventricles. We also investigated the relation between white and grey matter metabolites with neurological outcome and survival.

\section{Materials and methods}

\section{Patients}

This retrospective study was performed at the neonatal intensive care unit of the University Medical Center Groningen. From our medical records, we identified all term infants who were admitted because of clinical signs of hypoxic ischemic encephalopathy (HIE) and in whom MRI including MRS was performed, between January 2001 and July 2007. Perinatal asphyxia was defined by at least two of the following criteria: 1) signs of fetal distress, consisting of decelerations on fetal monitoring by cardiotocograph or meconium-stained amniotic fluid; 2) umbilical cord arterial $\mathrm{pH}$ less than 7.1 ; 3) Apgar scores less than 5 at 5 min after birth; and within days 4) multi organ failure syndrome; 5) neonatal HIE as described by Sarnat [19]. Exclusion criteria were: a gestational age below 36 weeks, presence of severe congenital malformations or diseases with neurological complications, and lack of clinical follow up examination.
Cerebral damage was evaluated by a combination of neurological examination, and amplitude integrated EEG. If outcome could not be predicted on these diagnostic tests, an additional MRI was performed to identify the extent of the cerebral damage following HIE. In the worst cases withdrawal of care was not based on the results of MRI and MRS, indicating that the selection of survivors/nonsurvivors was not biased by this. None of the infants were treated with therapeutic hypothermia, because this had not been introduced in our unit at the time of the study. The study was approved by the review board of the University Medical Center Groningen.

\section{MRS examination}

An 8-channel transmit/receive head coil of a 1.5T Magnetom Sonata system (Siemens AG, Erlangen, Germany) was used for proton (1H) MRS. 2D-chemical shift imaging (CSI) point resolved spectroscopy measurements were performed with a repetition time (TR) of $1500 \mathrm{~ms}$ and an TE of $135 \mathrm{~ms}$ resulting in T1- and T2-weighting of spectra [20]. A transverse T2-weighted fast spin-echo series was used as guidance for examining an approximately $5 \times 5 \times$ $2 \mathrm{~cm}^{3}$ supraventricular volume of interest, located cranial to the lateral ventricles, in a $16 \times 16$ phase encoded field of view of $16 \times 16 \mathrm{~cm}^{2}$ resulting in MRS voxels of $1 \times 1 \times 2 \mathrm{~cm}^{3}$ (7 min acquisition time) (Fig. 1). Since 2001, the standard brain MRI measurement protocol for infants following developmental delay and perinatal complications such as asphyxia has included MRS measurement according to the above specifications [21]. To obtain good quality spectra not affected by partial volume effects or by artefacts caused by movements near the eyes and ears we chose this particular volume of interest superior to the corpus callosum to measure a large transverse brain area containing both white and grey matter and little cerebrospinal fluid [22].

The standardised postprocessing protocol consisted of water reference processing, Hanning filtering (center $0 \mathrm{~ms}$, width $512 \mathrm{~ms}$ ), zero filling from 512 data points to 1024 data points, Fourier transformation, frequency shift correction, sixth order polynomial baseline correction, phase correction and frequency domain curve fitting. The curve fitting was set to fit peaks to Gaussian lineshapes, including the chemical shift ranges of 3.1-3.3 ppm for Cho, 2.9-3.1 for Cr, 1.9-2.1 for NAA, and 1.2-1.4 for Lact. The raw data were thus processed automatically, allowing for operator-independent quantification. The CSI voxels on the edge of the volume of interest (subject to signal drop-off) were deducted from the total data matrix. The inner 24 voxels were analysed and separated into the two central columns mainly containing grey matter (GM) (12 voxels) and the remainder of 12 voxels filled with white matter (WM) (Fig. 1), as described elsewhere [23]. 


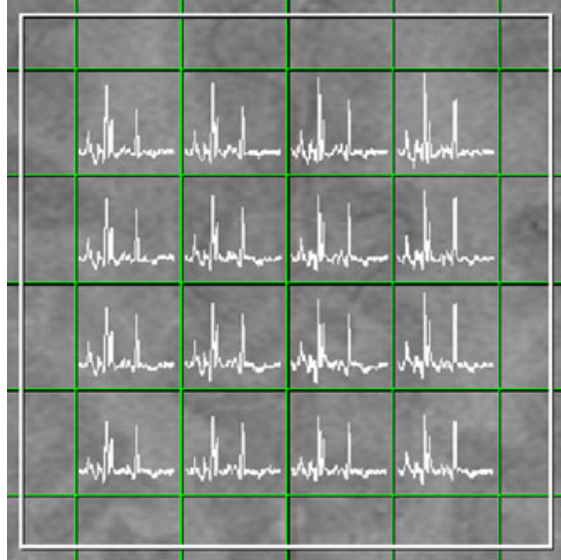

a

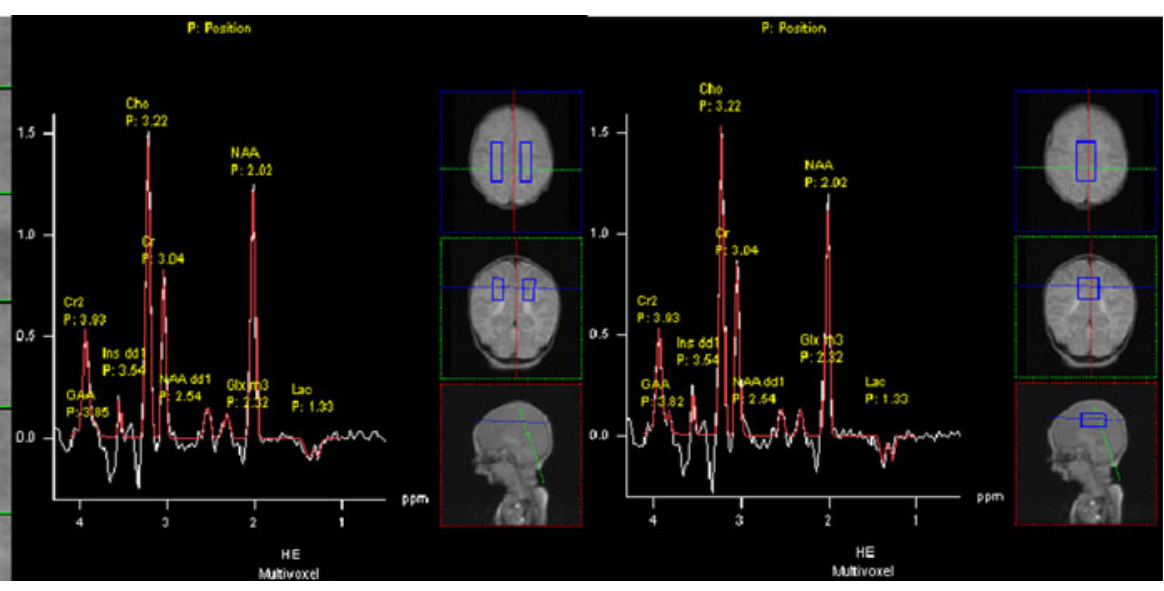

C

Fig. 1 MRS spectral map (a) and the summarised white matter and grey matter spectra (b, c) of a 7-day-old asphyxia patient with a normal neurologic outcome

The quantifications, also those of the small lactate peak in the summarised blocks of 12 or 24 spectra, were reliable and, therefore, no manual adjustments were performed. To limit patient examination times, absolute quantification requiring additional CSI measurements without water suppression was not performed. To facilitate tissue signal comparison, we expressed the GM and WM metabolites in percent (\%) of the summed peak areas of Cho, $\mathrm{Cr}$ and NAA in the 24 analysed voxels, a method used before [22, 24].

\section{Neurodevelopmental assessment}

Neurodevelopmental follow-up was performed at a minimum age of 18 months. Long term outcome was divided into four groups: 1) normal neurological outcome without complications; 2) minor neurological dysfunction; 3) severe neurological dysfunction; and 4) deceased. Severe neurological dysfunction was defined by a gross motor function classification system (GMFCS) [25] of three or more (not being able to walk independently). These children were treated in specialised rehabilitation centres, which performed systematic Bayleys developmental tests [26]. Children who were not treated systematically in specialised rehabilitation centres, were invited for follow-up examination. The neurological examination for toddler age by Hempel [27] was used till the age of 5 years. In two children who were older than 5 years we used the neurological examination by Touwen [28]. The examination was filmed and classified by a specialised paediatrician (C. v.d. V.) blinded for the clinical condition after birth and for the MRI and MRS results.

Statistical analysis

Statistical analysis was performed using SPSS version 17.0. A Kruskal Wallis test for non-parametric parameters was used to investigate significance between the four groups. Group-to-group differences were subsequently assessed by Mann-Whitney $U$-test. Differences between survivors $(n=$ 19) and non-survivors $(n=5)$ were assessed by MannWhitney $U$-test. A $P$ value of less than 0.05 was considered significant.

\section{Results}

A total of 26 infants admitted to the NICU were selected from our medical database. Two infants were excluded: one infant because of the coexistence of a severe congenital anomaly, and one infant because there was no long term follow-up. Therefore, the cohort of this study consisted of 24 infants (14 boys, 10 girls) with a median gestational age of $39^{3}$ (range from $36^{2}$ to $42^{0}$ ). Clinical characteristics of the study population are summarised in Table 1. MRI-MRS was performed at a median age of 7 (range 3 to 16) days after birth. At follow-up 11 children were normal, 5 had minor neurological dysfunction, 3 had severe neurological dysfunctions (all were diagnosed having a cerebral palsy with GMFCS of IV), and 5 infants died during the neonatal period.

The relative concentrations of the MRS metabolites in the four categories of neurological assessment are presented in Table 2. Both in the white and grey matter brain tissue NAA and Lact were significantly different between the four subgroups, Cho in the grey matter only. Compared to infants with normal outcome the infants that were severely neurologically abnormal or died had significant decreases of NAA and an increase of Lact.

MRS outcome in survivors $(n=19)$ and deaths within the neonatal period $(n=5)$ is shown in Table 3 . High levels of Cho and Lact in the grey matter differentiated non- 


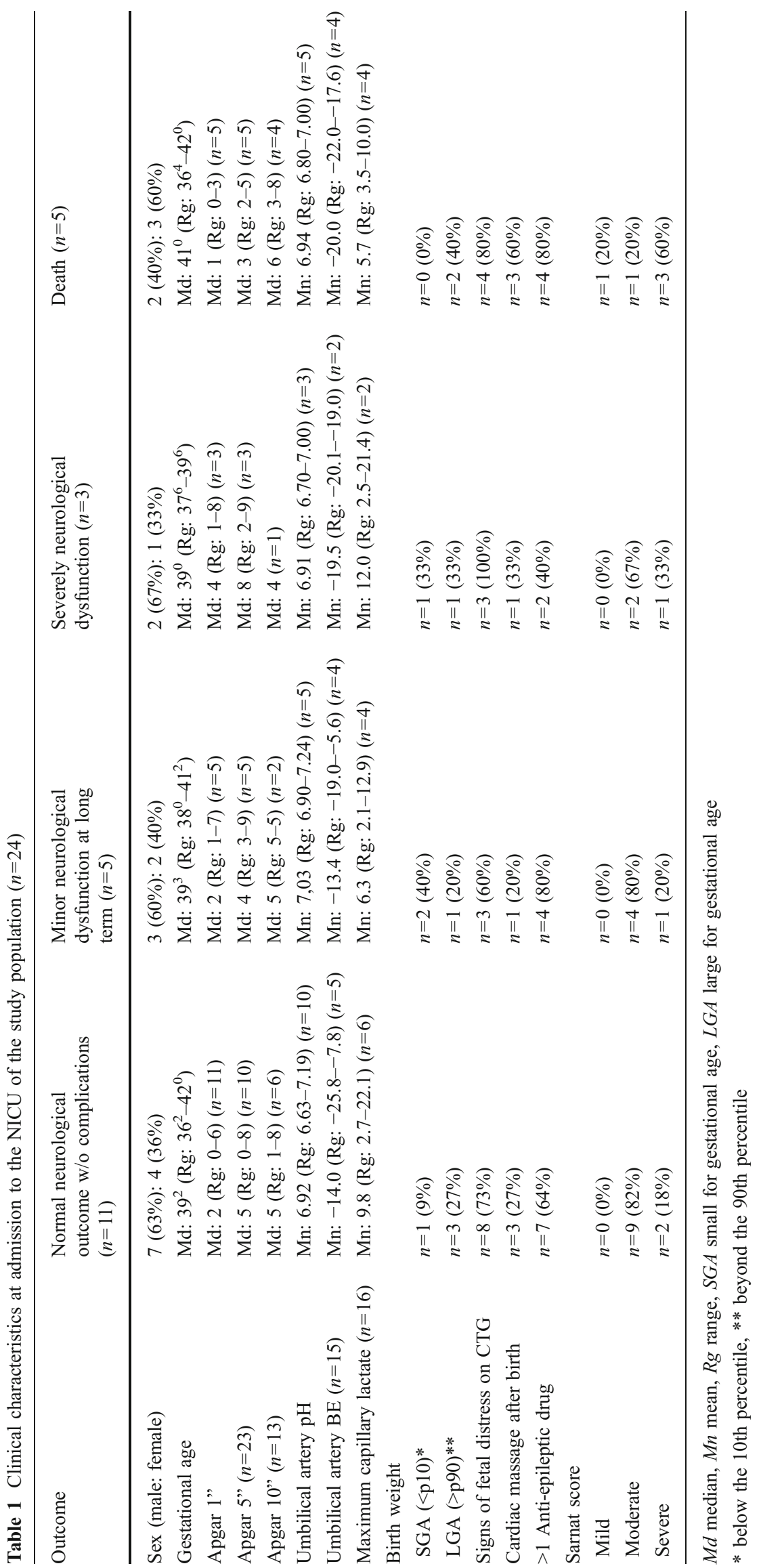


Table 2 Relative concentrations, in percent (\%) of the summed peak areas of $\mathrm{Cho}, \mathrm{Cr}$ and NAA in the volume of interest, of the major MRS detected brain metabolites in the 24 patients with perinatal asphyxia subdivided into the four categories of neurodevelopmental outcome at long term ( $>18$ months). Significant differences from normal outcome group are shown between brackets (Mann-Whitney $U$-test). Last column: Significance of the difference between the four groups

\begin{tabular}{|c|c|c|c|c|c|}
\hline & $\begin{array}{l}\text { Normal neurological } \\
\text { outcome w/o complications } \\
(n=11)\end{array}$ & $\begin{array}{l}\text { Minor neurological } \\
\text { dysfunction at long } \\
\text { term }(n=5)\end{array}$ & $\begin{array}{l}\text { Severe neurological } \\
\text { dysfunction }(n=3)\end{array}$ & Death $(n=5)$ & $\begin{array}{l}\text { Significance }(P) \text { of difference } \\
\text { between the } 4 \text { groups according } \\
\text { to Kruskal Wallis test }\end{array}$ \\
\hline \multicolumn{6}{|l|}{ WM } \\
\hline Cho & $49 \pm 4$ & $49 \pm 4$ & $52 \pm 3$ & $57 \pm 9$ & 0.140 \\
\hline $\mathrm{Cr}$ & $26 \pm 4$ & $24 \pm 2$ & $30 \pm 6$ & $25 \pm 4$ & 0.517 \\
\hline NA & $28 \pm 4$ & $28 \pm 4$ & $17 \pm 4(0.016)$ & $20 \pm 4(0.006)$ & 0.005 \\
\hline Lact & $6 \pm 4$ & $12 \pm 6(0.027)$ & $21 \pm 19$ & $14 \pm 8(0.027)$ & 0.039 \\
\hline \multicolumn{6}{|l|}{ GM } \\
\hline Cho & $46 \pm 3$ & $48 \pm 3$ & $48 \pm 4$ & $54 \pm 4(0.002)$ & 0.023 \\
\hline $\mathrm{Cr}$ & $25 \pm 2$ & $24 \pm 2$ & $31 \pm 6$ & $25 \pm 5$ & 0.190 \\
\hline NAA & $26 \pm 4$ & $28 \pm 3$ & $22 \pm 4$ & $20 \pm 5(0.047)$ & 0.031 \\
\hline Lact & $7 \pm 4$ & $9 \pm 6(0.047)$ & $15 \pm 13$ & $16 \pm 6(0.011)$ & 0.042 \\
\hline
\end{tabular}

survivors from survivors $(P=0.003$ and $P=0.017$, respectively). In the white matter tissue included in the volume of interest the differences in Cho and Lact were not significant, whereas NAA showed similar reductions in white and grey matter brain tisue $(P=0.025$, both). A representative result for an asphyxiated infant with a normal neurological outcome is shown in Fig. 1. In Fig. 2, an example of an infant that died, NAA level is very low and Lact very high as compared with the case represented by Fig. 1.

\section{Discussion}

In both the white and the grey matter brain tissue in a transverse volume of interest located cranial to the ventricles, NAA and Lact showed significant differences between four subgroups of neurological outcome following perinatal asphyxia (normal, minor neurological dysfunction, severe neurological dysfunction and death). In addition Cho was increased in the grey matter only, in case of death (Table 2). From the most favourable outcome to the worst Cho and Lact tend to increase by 8 or $9 \%$ of summed metabolites, whereas NAA drops by 6 to $8 \%$. Our findings for Lact and NAA are in good agreement with the recent meta-analysis of previous MRS studies of the thalamic or basal ganglia brain region indicating that amongst the various possible metabolite ratios Lact/NAA is the best biomarker for predicting adverse outcome after NE [1]. We conclude that the noted metabolite level changes in asphyxia probably reflect a phenomenon affecting the entire brain. However, in our data the differences between the various pairs of neurology subgroups are modest. For example, the second and third group of Table 2, minor neurological dysfunction and severe neurological dysfunction, only differ significantly in NAA (by $11 \%$ in the white matter and $6 \%$ in the grey matter; $P=0.025$, both).
Table 3 Relative concentrations, in per cent $(\%)$ of the summed peak areas of Cho, $\mathrm{Cr}$ and NAA in the volume if interest, of the major MRS detected brain metabolites in the 24 patients with perinatal asphyxia subdivided into death or survival

\begin{tabular}{lccc}
\hline & Survivors $(n=19)$ & Deaths $(n=5)$ & $\begin{array}{l}\text { Significance }(P) \text { of difference between the } \\
2 \text { groups according to Mann-Whitney } U \text {-test }\end{array}$ \\
\hline WM & & & \\
Cho & $49 \pm 4$ & $57 \pm 9$ & 0.060 \\
Cr & $26 \pm 5$ & $25 \pm 4$ & 0.594 \\
NAA & $26 \pm 5$ & $20 \pm 4$ & 0.025 \\
Lact & $10 \pm 9$ & $14 \pm 8$ & 0.110 \\
GM & & & 0.003 \\
Cho & $47 \pm 3$ & $54 \pm 4$ & 0.749 \\
Cr & $26 \pm 4$ & $25 \pm 5$ & 0.025 \\
NAA & $26 \pm 4$ & $20 \pm 5$ & 0.017 \\
Lact & $9 \pm 6$ & $16 \pm 6$ & \\
\hline
\end{tabular}




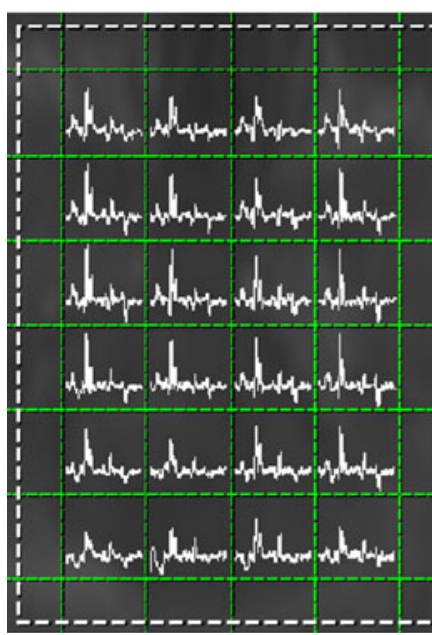

a

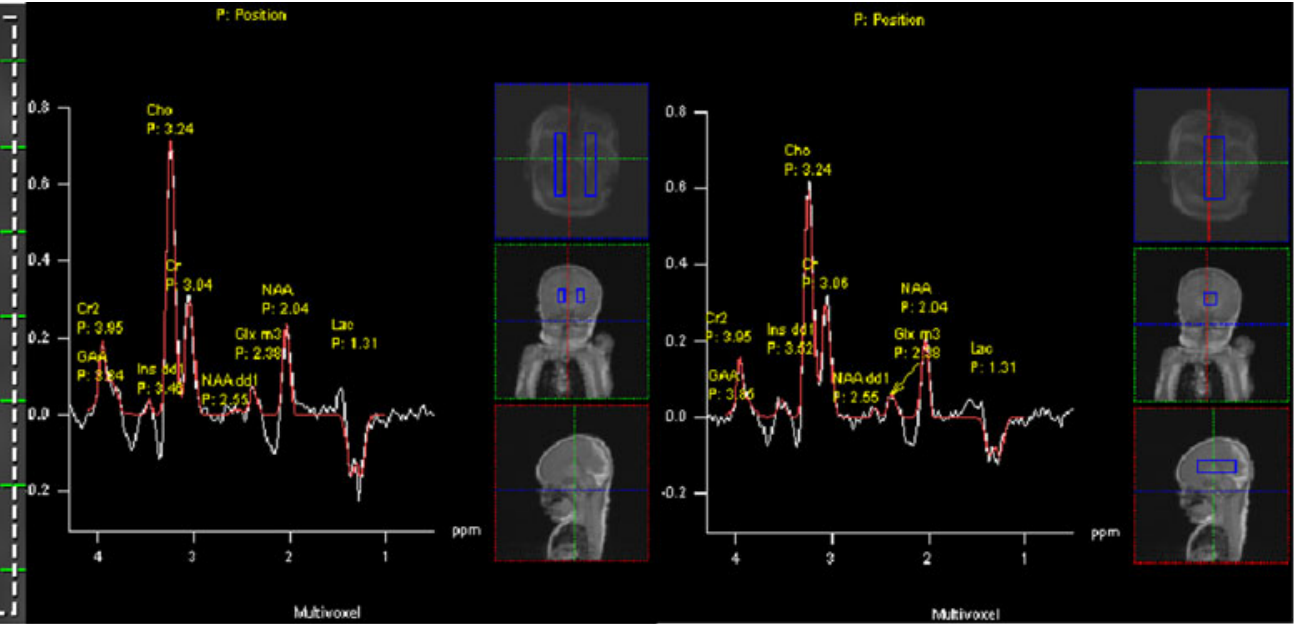

b

C

Fig. 2 MRS spectral map (a) and the summarised white matter and grey matter spectra (b, c) of an 8-day-old asphyxia patient who died 2 days later

In addition to investigating the relationship between brain metabolism and neurological outcome, the purpose of this study also was to compare MRS quantified brain metabolism to survival. Compared to the 19 survivors, the five infants that died following perinatal asphyxia, had significantly increased levels of Cho and Lact, $+7 \%$ for both metabolites, in the grey matter only (Table 2). Our second conclusion is therefore that while quantitative MRS performed between days 3 to 16 after birth offers little differentiation between groups of asphyxiated infants categorised in terms of neurological outcome, the findings in the grey matter brain tissue have a higher predictive value than those in the white matter tissue as far as survival is concerned. Previous comparisons of survivors vs. fatal outcome indicated generally increased basal ganglia Lact/ NAA and Lact/Cr ratios $[8,12,14,29,30]$ or increased Lact/Cho ratios [31] in the latter category. So, the grey matter Lact concentrations increases observed in this study fit with the basal ganglia observations in the above studies. The present study, however, appears to be the first showing increased grey matter Cho levels in asphyxiated infants with fatal outcome.

The elevation of Lact in the brain spectra of asphyxiated infants is obvious, being a universal phenomenon of hypoxia and anaerobic glycolysis in any human tissue. However, the increase of Cho in the grey matter brain tissue of infants with a fatal outcome, is less easily explained. In general, the level of Cho in infants reflects the degree of dysmyelination and decreases steadily during the proces of myelination during the first years of life [21]. The observed Cho elevation may thus be interpreted as reflecting deficient development of the neurons.

One limitation of this study is the spread of neonatal age, 3 to 16 days after birth, at which the MRS was performed.
Measurement at a later time after the onset of asphyxia may have led to tissue loss leading to further going NAA decreases and Lact increases as compared with infants examined earlier. Another limitation of the study is that we did not include controls. In our institution, as probably anywhere else, entirely normal infants are not examined by MRI during the first month after birth. Another issue that could be considered a limitation in this study is that we studied a transverse plane cranial to the ventricles rather than the basal ganglia region. Our motivation for this is twofold, firstly the better quality of MR spectra measured in the supraventricular plane as compared to a plane through the basal ganglia [21, 24], secondly because in this plane the virual lack of cerebrospinal fluid facilitates quantitative comparisons between voxels [23]. In our study the use of a TE of $135 \mathrm{~ms}$, optimal for the unambiguous detection of Lact, may have prohibited us from reproducing the decreases in the minor signals of glutamate/glutamine, amino acids with short $\mathrm{T} 2$ relaxation times, previously observed by Angeles et al [18].

This study was limited to the evaluation of the results of MR spectroscopy alone in the assessment of asphyxia. Our findings should, however, not be interpreted as evidence that MRS can replace imaging. The literature to date suggests that in clinical practice MRS can become a useful adjunct to conventional MRI. At our institution analyses and follow-ups are currently underway to compare the diagnostic value of MRI and MRS, especially to look at the added value of MRS in those cases where the results of MRI alone were ambiguous.

In conclusion, the levels of Cho, NAA and Lact in both white and grey matter brain tissue are altered in perinatal asphyxia. New in our study is that, the levels of Cho and Lact measured in the grey matter appear to be the most 
important indicators survival. It is therefore advised to include grey matter brain tissue in the region of interest examined by multivoxel MR spectroscopy to help predict clinical outcome in infants suffering from perinatal asphyxia.

Open Access This article is distributed under the terms of the Creative Commons Attribution Noncommercial License which permits any noncommercial use, distribution, and reproduction in any medium, provided the original author(s) and source are credited.

\section{References}

1. Thayyil S, Chandrasakaran M, Taylor A et al (2010) Cerebral magnetic resonance biomarkers in neonatal encephalopathy: a meta-analysis. Pediatrics 125:e382-e395

2. Dubowitz LM, Bydder GM (1985) Nuclear magnetic resonance imaging in the diagnosis and follow-up of neonatal cerebral injury. Clin Perinatol 12:243-260

3. Peden CJ, Rutherford MA, Sargentoni J, Cox IJ, Bryant DJ, Dubowitz LMS (1993) Proton spectroscopy of the neonatal brain following hypoxic ischaemic injury. Dev Med Child Neurol 35:502-510

4. Hanrahan JD, Sargentoni J, Azzopardi D et al (1996) Cerebral metabolism within 18 hours of birth asphyxia: a proton magnetic resonance spectroscopy study. Pediatr Res 39:584-590

5. Penrice J, Cady EB, Lorekk A, Wylezinska M et al (1996) Proton spectroscopy of the brain in normal preterm and term infants, and early changes after perinatal hypoxia-ischemia. Pediatr Res 40:6-14

6. Holshouser SA, Ashwal S, Gy L et al (1997) Proton spectroscopy after acute central nervous system injury; outcome prediction in neonates, infants, and children. Radiology 202:487-496

7. Barkovich AJ, Baranski K, Vigneron D et al (1999) Proton MR spectroscopy for the evaluation of brain injury in asphyxiated, term neonates. AJNR Am J Neuroradiol 20:1399-1405

8. Amess PN, Lorek A, Townsend J et al (1999) Early brain proton magnetic resonance spectroscopy and neonatal neurology related to neurodevelopmental outcome at 1 year in term infants after presumed hypoxic-ischaemic brain injury. Dev Med Child Neurol 41:436-445

9. Robertson NJ, Lewis RH, Cowan FM et al (2001) Early increases in brain myo-inosotol measured by proton magnetic resonance spectroscopy in term infants with neonatal encephalopathy. Pediatr Res 50:692-700

10. Groenendaal F, Roelants-van Rijn AM, van der Grond J, Toet MC, de Vries LS (2001) Glutamate in cerebral tissue of asphyxiated neonates during the first week of life demonstrated in vivo using proton magnetic resonance spectroscopy. Biol Neonate 79:254-257

11. Fan G, Wu Z, Chen L, Guo Q, Ye B, Mao J (2003) Hypoxic-ischemic encephalopathy in full-term neonate: correlation of proton MR spectroscopy with MR imaging. Eur J Radiol 45:91-98

12. Brissaud O, Chateil J-F, Bordessoules M, Brun M (2005) Chemical shift imaging and localized magnetic resonance spectroscopy in full-term asphyxiated neonates. Pediatr Radiol 35:998-1005

13. Shanmugalingam S, Thornton JS, Iwata O et al (2006) Comparative prognostic utilities of early quantitative magnetic resonance imaging spin-spin relaxometry and proton magnetic resonance spectroscopy in neonatal encephalopathy. Pediatrics 118:1467-1477
14. Zhu W, Zhong W, Qi J, Yin P, Wang C, Chang L (2008) Proton magnetic resonance spectroscopy in neonates with hypoxicischemic injury and its prognostic value. Transl Res 152:225-232

15. Cady EB (1996) Metabolic concentrations and relaxation in perinatal cerebral hypoxic-ischemic injury. Neurochem Res 9:1043-1052

16. Boichot C, Walker PM, Durand C et al (2006) Term neonate prognosis after perinatal asphyxia: contributions of MR imaging, MR spectroscopy, relaxation times, and apparent diffusion coefficients. Radiology 239:839-848

17. Cheong JLY, Cady EB, Penrice J, Wyatt JS, Cox IJ, Robertson NJ (2006) Proton Mr spectroscopy in neonates with perinatal cerebral hypoxic-ischemic injury: metabolic peak-area ratios, relaxation times, and absolute concentrations. AJNR Am J Neuroradiol 27:1546-1554

18. Angeles DM, Ashwal S, Wycliffe ND et al (2007) Relationships between opioid therapy, tissue damaging procedures, and brain metabolites as measured by proton MRS in asphyxiated term neonates. Pediatr Res 61:614-621

19. Sarnat HB, Sarnat MS (1976) Neonatal encephalopathy following fetal distress. A clinical and electroencephalographic study. Arch Neurol 33:696-705

20. Sijens PE, van den Bent MJ, Nowak PJ, van Dijk P, Oudkerk M (1997) $1 \mathrm{H}$ chemical shift imaging reveals loss of brain tumor choline signal after administration of Gd-contrast. Magn Reson Med 37:222-225

21. Verbruggen KT, Maurits NM, van Spronsen FJ, Brouwer OF, Sijens PE (2009) Quantitative multivoxel proton spectroscopy of the brain in developmental delay. J Magn Reson Imaging 30:716-721

22. Sijens PE, Oudkerk M, de Leeuw FE et al (1999) $1 \mathrm{H}$ chemical shift imaging of the human brain at age 60-90 years reveals metabolic differences between women and men. Magn Reson Med 42:24-31

23. Sijens PE, Mostert JP, Oudkerk M, De Keyser J (2006) 1H MR spectroscopy of the brain in multiple sclerosis subtypes with analysis of the metabolite concentrations in gray and white matter: initial findings. Eur Radiol 16:489-495

24. van Oostrom JC, Sijens PE, Roos RA, Leenders KL (2007) $1 \mathrm{H}$ magnetic resonance spectroscopy in preclinical Huntington disease. Brain Res 1168:67-71

25. McDowell B (2008) The gross motor function classification system-expanded and revised. Dev Med Child Neurol 50:725730

26. Bayley N (1993) Bayley Scales of Infant Development, Second Edition. San Antonio, TX: The Psychological Corporation; www. psychocorp.com

27. Hempel MS (1993) The neurological examination for toddler-age [dissertation]. Groningen University Press, Groningen

28. Touwen BC (1968) Neurological examination of the young child. Ned Tijdschr Geneeskd 112:1112

29. Hanrahan JD, Cox IJ, Azzopardi J et al (1999) Relation between proton magnetic resonance spectroscopy within 18 hours of birth asphyxia and neurodevelopment at 1 year of age. Dev Med Child Neurol 41:76-82

30. L'Abee CL, de Vries LS, van der Grond JS, Groenendaal F (2005) Early diffusion-weighted MRI and $1 \mathrm{H}$ magnetic resonance spectroscopy in asphyxiated full-term neonates. Biol Neanate 88:306-312

31. Zariff MK, Astrakas LG, du Poussant TY, Plessis A, Zurakowski D, Tzika AA (2002) Prediction of adverse outcome with cerebral lactate level and apparent diffusion coefficient in infants with perinatal asphyxia. Radiology 225:859-870 